\title{
GENERIC OBJECT RECOGNITION USING FORM \& FUNCTION
}




\section{SERIES IN MACHINE PERCEPTION AND ARTIFICIAL INTELLIGENCE*}

Editors: H. Bunke (Univ, Bern, Switzerland)

P. S. P. Wang (Northeastem Univ., USA)

Vol. 10: Generic Object Recognition Using Form and Function

(Eds. K. W. Bowyer and L. Stark)

Vol. 11: Experimental Environments for Computer Vision and Image Processing (Eds. H. I. Christensen and J. L. Crowley)

Vol. 12: Computing Conceptual Organization in Computer Vision

(Eds. S. Sarkar and K. L. Boyer)

Vol. 13: Progress in Automatic Signature Verification

(Eds. R. Plamondon)

Vol. 14: Document Analysis Systems

(Eds. A. Dengel and L. Spitz)

Vol. 15: Parallel Image Analysis and Processing (Eds. K. Inove, A. Nakamura, M. Nivat, A. Saoudi and P. S. P. Wang)

Vol. 16: Document Image Analysis

(Eds. H. Bunke, P. S. P. Wang and H. Baird)

Vol. 17: Applications of Al, Machine Vision and Robotics (Eds. K. L. Boyer, L. Stark and H. Bunke)

Vol. 18: VLSI and Parallel Computing for Pattem Recognition and $A I$ (Ed. N. Ranganathan)

Vol. 19: Parallel Image Analysis: Theory and Applications

(Eds. L. S. Davis, K. Inoue, M. Nivat, A. Rosenfeld and P. S. P. Wang)

Vol. 20: Picture Interpretation: A Symbolic Approach

(Eds. S. Dance, T. Caelli and Z.-Q. Lin)

Vol. 21: Modelling and Planning for Sensor Based Intelligent Robot Systems (Eds. H. Bunke, T. Kanade and H. Noltemeier)

\section{Forthcoming}

Vol. 22: Machine Vision for Advanced Production (Eds. M. Pietikainen and L. F. Pau)

Vol. 23: Machine Learning and Perception (Eds. G. Tasini, F. Esposito, V. Roberto and P. Zingarett)

"For the complete list of titles in this series, please write to the Publisher. 


\title{
GENERIC OBJECT RECOGNITION USING FORM \& FUNCTION
}

\author{
Louise Stark \\ University of the Pacific \\ Stockton, Califomia, USA \\ Kevin Bowyer \\ University of South Florida \\ Tampa, Florida, USA
}




\section{Published by}

World Scientific Publishing Co Pte Ld

P O Box 128, Farrex Raad, Singapore 912805

USA office: Suite IB, 1060 Mrin Street, River Edge, NJ 07661

UK office: 57 Shelton Street, Covent Garden, London WC2H 9HE

\section{GENERIC OBJECT RECOGNITION USING FORM AND FUNCTION}

Copyright 11996 by World Scientific Publishing Co. Pte. Ld.

All rights reserved. This book, or parts the reof, may not be reproduced in any form or by any means. electronic or mechanical, including photocopying, recording or any information storage and retrieval system now brown or to be invented, without written permission from the Publister.

For photocopying of material in this volume, please pay a copying foe through the Copyright Clearance Center, Inc., 222 Rosewood Drive, Danvers, MA 01923. USA.

ISBN 981-02-1508-8

Printed in Singapore by Uto-Print 


\title{
Dedication
}

\author{
To Ken \\ from Louise \\ To Susan, Mathew and Jimmy \\ from Kevin
}


This page is intentionally left blank 


\section{Preface}

Initial work on the Grupr rescarch project began in the summer of 1987. This monograph provides a detailed record of the results of the Gaurr (Generic Recognition Using Form and Function) project through 1994. The goal of the project is to develop techniques for vision systems to recognize an object by reasoning about what function(s) the object might serve.

The major effort to date has been the development and evaluation of a sequence of systems which analyze a 3-D shape description of an unknown object. Some of the more important contributions in this part of the project are (a) the first working demonstration of the feasibility of the "form and function" approach to reasoning about 3-D shapes, (b) verification of the hypothesis that a small number of knowledge primitives could be used as building blocks to define a broad range of object categories, and (c) the development of an indexing mechanism to make processing for recognition more efficient without any substantial decrease in correctness of classification. Results from this portion of the project are given for the analysis of over 4503 - D shape descriptions created with a solid modeling tool.

We have also developed and demonstrated a version of the Grurr system which reasons about partial 3-D shape information of the type that can be derived from one view of an object, or from some secpuence of views which does not allow acquisition of a complete shape model. This line of research gave rise to the Opus ("Object Plus Unseen Space") shape model, and also to strategies for selecting a sequence of views of an object. Results from this portion of the project are given for the analysis of over 200 shape descriptions extracted from range images.

The domain of competence for the initial versions of GRuFr was restricted to rigid 3-D shapes. We have recently generalized this to allow articulated 3-D shapes; that is, objects composed of rigid parts with connections between them.

Lastly, while shape properties are important, verification of actual functionality generally requires consideration of properties beyond pure static shape. Initial reasoning about the static 3 -D shape provides a labeling of the object structure that can 
be used to guide interactions that lead to confirming or rejecting the suggested object function. A system has been implemented which provides a simulated environment to test the generation and execution of a plan for interaction.

The initial version of the Grury system was created by one of the authors, Louise Stark, as part of her Ph.D. dissertation in the College of Engineering at the University of South Florida. A number of people have since collaborated with the authors on different aspects of the project. These people include Natalie Bennett, Barry Bruno, Diane Cook, David Eggert, Dmitry Goldgof, Kevin Green, Larry Hall, Adam Hoover, Haihua Liuh, Melanie Sutton and Kevin Woods. In order to recognize all of these people's different contributions, many chapters of this book have a separate list of co-authors.

Support for the Grurr project and the students working on the project has come from a variety of sources. The primary support has been grants from the Air Force Office of Scientific Research and the National Science Foundation.

- AFOSR F49620-92-J-0223,

- NSF IRI-9120895,

- AFOSR.89-0036, and

- UES/AFOSR Grant S-760-00MG-003.

Graduate students working on the project have also been supported by a Patricia Harris Fellowship and NASA Florida Space Grant Consortium fellowships. Undergraduate students supported by a sequence of NSF Research Experience for Undergraduates grants have also made contributions to the Grorr project:

- NSF CDA-92-00369,

- NSF CDA-91-00898, and

- NSF CDA-90-00802.

In addition, Oak Ridge National Laboratories (ORNL) has provided support for this project through allowing us to use the facilities of the CESAR Lab, in particular the HERMIES-3 mobile robot and laser range finder used in some of our experimental work. 
The Gaurr project is still ongoing and active. Copies of the most recent publications related to the GuUrr project are generally available by anonymous ftp to figment.csee.usf.edu from the directory pub/Gruff-papers. Further information can be also be accessed via the URL http://marathon.csee.usf.edu/. In general, all of the 3-D shape models and the raw images used in published work are made available to interested researchers through the WWW site. 
This page is intentionally left blank 


\section{Acknowledgments}

We would like to thank the CESAR lab at Oak Ridge National Labs, especially Judd Jones, Ole Henry Dorum, Frank Sweeney and Reinhold Mann, for making it possible for us to acquire images with the Odetics LRF mounted on the HERMIES III mobile robot system.

We would like to thank Stan Sirlaroff and Sandy Pentland at the MIT Media Lab for their assistance in making use of the Thing Wornd system.

Portions of the material in this book have been re-used with permission from the following sources:

- Generic recognition of articulated objects through reasoning about potential function. Green, K., Eggert, D., Stark, L. and Bowyer, K.W. Computer Vision and Image Understanding, 62 (2), (September 1995), 177-194.

- GRUFF-3: Generalizing the domain of a function-based recognition system. Sutton, M., Stark, L. and Bowyer, K. Pattern Recognition, 27 (12), (December $1994), 1743 \cdot 1766$.

- Stark, L. Recognizing object function through reasoning about 3-D shape and dynamic physical properties, Compuler Vision and Pattern Recognution, Seattle, Washington, (June 1994), 546.553.

- Function based generic recognition for multiple object categories. Stark, L. and Bowyer, K.W. CVGIP: Image Understanding, 58 (1), (January 1994). 1.21.

- Function-based object recoguition from incomplete knowledge of object shape. Stark, L., Hoover, A.W.. Goldgof, D.B. and Bowyer, K.W. IEEE Workshop on Qualitative Vision, New York (June 1993), 11.22.

- Function based generic recognition for multiple object categories, Sutton, M., Stark, L. and Bowyet, K.W. in Three-dimensional Object Recognition Systems, A.K. Jain and P.J. Flynn, cditors, Elsevier Science Publishers, 1993, 447.470. 
- Stark, L., and Bowyer, K.W. Indexing function-based categories for generic object recognition, CVGIP: Image Understanding, 59 (1), (January 1994), 121.

- Achieving generalized object recognition through reasoning about association of function to structure, Stark, L., and Bowyer, K.W. IEEE Transactions on Pattern Analysis and Machine Intelligence, 3 (10), (October 1991) 1097-1104. 


\section{Table of Contents}

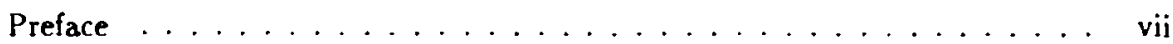

Acknowledgements ........................ xi

I Introduction .................... 1

1.1 Function-Based Versus Model-Based ............. 2

1.2 Organization of the Book ............... 5

Il Related Work ..................... 7

2.1 Other Directions Toward Generic Vision . . . . . . . . . . . 7

2.1.1 Parameterized 2-D feature configurations . . . . . . . . 7

2.1.2 Parameterized 3-D geometric models . . . . . . . . . . 9

2.1.3 Parameterized 3-D structural models . . . . . . . . . 10

2.2 Kelated Work in Function-Based Recognition . . . . . . . . . 12

2.3 Summary ..................... 16

III The "Knowledge Primitives" ................ 17

3.1 Relative Orientation ................. 18

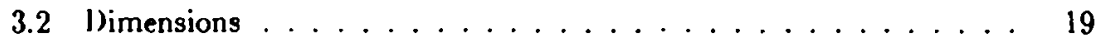

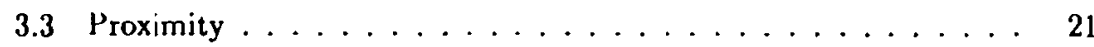

3.4 Stability........................ 22

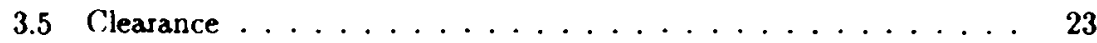

3.6 Enclosure ........................... 24

3.7 Evaluation Measures for the Primitives ............ 25

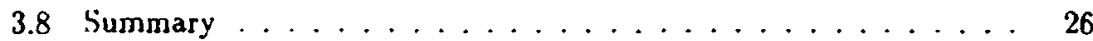




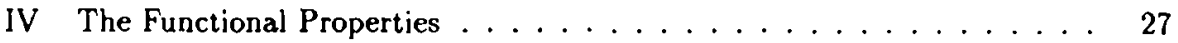

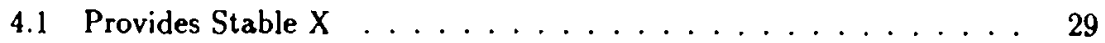

4.2 Provides X Surface . . . . . . . . . . . . . . . . 30

4.3 Provides $X$ Containment . . . . . . . . . . . . . . 31

4.4 Provides X Handle . . . . . . . . . . . . . . . . . . 32

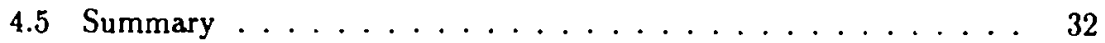

V The "Category Definition Tree" ................ 33

5.1 Structure of the Category Definition Tree . . . . . . . . . 33

5.2 Category Definition Tree as a Control Structure . . . . . . . . . 39

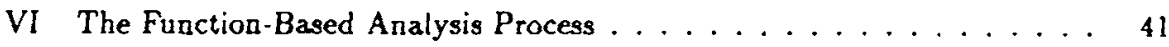

6.1 Measures used in the Process . . . . . . . . . . . . . 42

6.2 System Evaluation Process . . . . . . . . . . . . . . . . 44

6.2.1 Pre-processing the object shape . . . . . . . . . . 44

6.3 Recognition Stage . . . . . . . . . . . . . . 45

6.3.1 Rank order possible categories by indexing measure . . . . 45

6.3.2 Analyze shape . . . . . . . . . . . . . 48

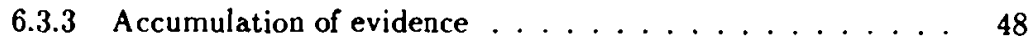

6.3 .4 Recognition output . . . . . . . . . . . . . . . . 50

6.4 Evaluation of Example Input Shape . . . . . . . . . 50

VII Recognition Results for Complete 3.D Shapes . . . . . . . . . . . 61

7.1 GRUFF Project History . . . . . . . . . . . . . 6 61

7.1 .1 Summary of results . . . . . . . . . . . . . 63

7.2 Beyond complete static 3 -D shape analysis . . . . . . . 65

VIII Function-Based Analysis using Partial Shape . . . . . . . . . . . 67

8.1 Reasoning with Partial Shape Descriptions . . . . . . . . . . 67

8.1.1 Acquisition of "Object Plus Unseen Space" models . . . . 68

8.1.2 Function-based recognition of OPUS models . . . . . . . 72

8.1.3 Summary of recognition results for the OPUS models . . . . 76 
IX Reasoning About Articulated Shapes ............... 81

9.1 Sequences of Shape Instances and Articulated Shapes . . . . . . . 81

9.2 Algorithm for Recovering Articulated Shapes . . . . . . . . . . . 85

9.2.1 Feature correspondence .............. 85

9.2.2 Part boundary representations . . . . . . . . . 86

9.2 .3 Connections .................. 86

9.2 .4 Linkage relations ................ 87

9.3 Function-Based Representation of Articulated Objects . . . . . . . 88

9.4 lunction-Based Interpretation of an Articulated Shape . . . . . . . 89

9.4.1 Processing for opposing_finger_grasp .......... 91

9.4.2 Processing for opposing_cutting_blartes . . . . . . . . . . 94

9.4.3 Processing for closable grasp_that_causes_cutting . . . . 9 96

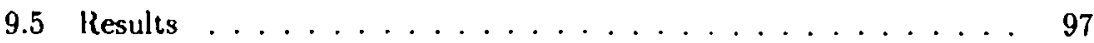

9.5.1 Acquisition of articulated shape models . . . . . . . . . 97

9.5.2 Categorization of articulated shape rnodels . . . . . . . 101

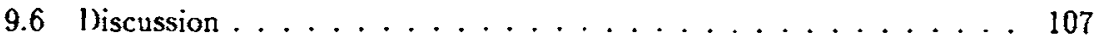

X Planned Interactions to Verify Functionality ............. 109

10.1 Knowledge Primitives for Interaction with an Object . . . . . . 110

10.2 Planned Interaction to Verify Functionality . . . . . . . . . . 112

10.2.1 Function verification plans executed in ThingWorld . . . 116

10.3 Summary ...................... 123

XI Future Directions ....................... 125

REFERENCES . . . . . . . . . . . . . . . . . . . 127

INDEX ......................... 134 